\title{
MODELO DE EVALUACIÓN DE LA CALIDAD DE CURSOS FORMATIVOS IMPARTIDOS A TRAVÉS DE INTERNET
}

(ASSESSMENT MODEL OF TRAINING PROGRAMMES BASED IN INTERNET)

Inmaculada Tello Díaz-Maroto

Escuela Universitaria Cardenal Cisneros (España)

\section{RESUMEN}

Debido al progresivo aumento de la oferta y la demanda de programas formativos a través de Internet, se hace absolutamente imprescindible para la selección adecuada de los mismos, la posibilidad de evaluar la calidad de dichos cursos formativos. Por ello, en la investigación llevada a cabo, se han analizado los modelos de evaluación existentes en la educación y se propone un modelo de evaluación de los programas formativos impartidos a través de Internet (MEPFI), el cual se encuentra incluido en un ciclo sistémico de diseño, creación, aplicación, evaluación y modificación de la formación. Se ha aplicado el modelo a una muestra de sujetos que siguen una serie de cursos formativos, analizando así la efectividad del modelo y las conclusiones a las que nos permite llegar.

Palabras clave: formación on-line, formación semipresencial, formación a distancia, calidad educativa, formación del profesorado, TIC, Internet, evaluación, calidad.

\begin{abstract}
Due to the increase in the supply and demand of training programmes based in Internet, it is vital they can be subjected to quality evaluation. Therefore, a research has been carried out regarding the assessment models used in education to define an assessment model of training programmes based in Internet (in Spanish MEPFI). The model proposed follows the principles of design, creation, implementation, evaluation and modification systemic cycle applied to training. It has been applied to a sample of students participating in some of these programmes in order to analyse the effectiveness of the model and to draw some conclusions.
\end{abstract}

Keywords: on-line learning, distance learning, onsite learning, blended learning, education quality, teacher training, TIC, Internet, evaluation, quality. 
"Es difícil preguntarnos si los entornos de aprendizaje abiertos y flexibles basados en las TIC nos reportan una educación cualitativamente mejor, más efectiva y más eficiente, y sobre todo cómo deben ser abordados estos nuevos modelos educativos desde un punto de vista pedagógico".

(Friedrich Scheuermann y Mario Barajas, 2003)

La evaluación de la calidad en la educación y la formación debe aplicarse también a las nuevas metodologías de enseñanza y aprendizaje, entre ellas, las TIC en general e Internet en particular. Pero como señalan Scheuermann y Barajas (2003), no basta con un estudio cuantitativo, sino que es preciso un estudio cualitativo de dichas enseñanzas, que nos aporte diversas perspectivas de mejora de los usuarios, para poderlas tener en cuenta en el rediseño de los cursos.

Ambos autores también mencionan la importancia de evaluar la eficiencia, efectividad y aspectos pedagógicos de dichos cursos ofertados en la red. Nosotros además, proponemos evaluar otros aspectos de los cursos que resultan de gran importancia para medir la calidad de los mismos, formulando un modelo de evaluación de la calidad de cursos formativos impartidos a través de Internet.

\section{TEORIZACIÓN DEL MODELO}

A partir de toda la revisión bibliográfica realizada y del análisis de los instrumentos de medida localizados en relación a la evaluación de programas formativos on-line, software educativo y páginas Web, surge la idea de diseñar un modelo de evaluación para valorar la calidad de los cursos de formación ofertados a través de Internet, con el fin de mejorar la oferta educativa ofertada a través de dicho medio.

Entre toda la bibliografía localizada y analizada para centrar el marco teórico de la investigación, cabe destacar el exhaustivo análisis llevado a cabo de la filosofía de Gestión dela Calidad Total, delas normas ISO 9001 y del Modelo Europeo de Excelencia Empresarial EFQM; adaptándolo al ámbito educativo y aún más concretamente, a la formación on-line, que es el objetivo de evaluación de la presente investigación.

Entre los instrumentos evaluados en este estudio, podemos mencionar:

- Esquema de Woerner, Rivers y Vockell (1991)

- Esquema de la Universidad del Sur de Florida (Pritchard, Micceri y Barret, 1989) 
- Esquema de Galvis, Prieto y Hernández (1986):

- Cuestionario C.E.S.E. (Medina, 1990)

- Herramienta de evaluación de patrocinio y Leote (1993)

- Propuesta de evaluación semiótica de Tucker (1992)

- Instrumento de evaluación de Software educativo de Martínez Ruizy Sauleda (1993)

- Instrumento de evaluación del software educativo de Aguirre y Marín (1994)

- Análisis del software educativo propuesto por el Departamento de Educación, Cultura y Deporte del Gobierno de Navarra

- Guía de evaluación del software educativo propuesto por el Grupo Orixe

- Criterios de calidad propuestos por Pere Marqués (1999)

- Evaluación de la Calidad de los materiales y recursos didácticos en el programa de formación on-line de directivos de instituciones educativas (2002)

- Evaluación de medios informáticos: Una escala de evaluación para software educativo (Barroso, Medel, Valverde, 1997).

- Ejemplo de Evaluación de software educativo multimedia. (Gómez Del Castillo, 1997).

- Ficha de evaluación de programas informáticos educativos. (GarcíaVarcángel).

Casi todos los instrumentos analizados mezclan valoraciones realizadas desde los "expertos-profesores" en una vertiente más técnico-pedagógica, con las de los alumnos en formación, con una vertiente más orientada hacia satisfacción con el programa. Deberíamos partir de la base de que un programa educativo no sólo debería incluir estos aspectos, sino también los relativos a eficacia, es decir, a los aprendizajes de los alumnos entendidos como resultados del programa. La dificultad de medir estos aspectos o la perspectiva de los procesos de enseñanza-aprendizaje desde el punto de vista del profesor o creador y no tanto desde el alumno, ha hecho que los instrumentos desarrollados hasta el momento no incluyan esta orientación. Pero en una era pedagógica marcada por una orientación del proceso centrada en el alumno, como va a ser la defendida por el Espacio Europeo de Educación Superior (EEES), es imposible hacer ninguna propuesta que no recoja esta orientación.

Por tanto, para la evaluación de la calidad de los programas formativos a través de la red, habrá que tener en cuenta parámetros específicos y novedosos, fundamentados en modelos experimentales que consideren tanto el diseño, como 
la implementación y los resultados de los mismos. De entre todos los modelos de evaluación de programas, aquel que puede aplicarse de manera más adecuada a esta orientación, es el desarrollado por Kirkpatrick $\left(1944^{1}\right)$. Para este autor, la evaluación de programas se basaría en cuatro niveles de análisis: Reacción, Aprendizaje, Conducta y Resultados. Veamos a continuación qué significa cada uno de ellos y su aplicación en esta investigación:

- Reacción: como indica la propia palabra, la evaluación en este nivel mide cómo reaccionan los participantes ante la acción formativa. Sería lo mismo que medir la satisfacción del cliente. Si el aprendizaje tiene que ser eficaz, es importante que los participantes reaccionen favorablemente hacia él. De otro modo, no estarán motivados para aprender.

En la investigación llevada a cabo, se ha diseñado un instrumento de medición que evalúa este nivel de la evaluación, el cual consiste en preguntar a los alumnos de los programas formativos sobre el grado en el que el curso ha resuelto sus expectativas (gusto y aceptación); es decir, comprobar la satisfacción que sobre diferentes aspectos del mismo tiene el alumno.

- Aprendizaje: se puede definir como el grado en que los participantes cambian actitudes, amplían conocimientos y/o mejoran habilidades, como consecuencia de asistir a una acción formativa.

Este nivel de la evaluación ha sido tenido en cuenta en la investigación a través de información proporcionada por la dirección de cada curso, a través de la cual podemos valorar la adquisición de los conocimientos, habilidades y procedimientos que el alumno ha conseguido aprender o desarrollar con la realización del curso a través de Internet. En terminología del EEES, se trataría de evaluar el desarrollo competencial del alumno, si por competencia (profesional) entendemos un aspecto complejo del aprendizaje del alumno fruto de la unión de tres factores: conocimientos, destrezas y actitudes; que forman al alumno de tal manera que sea capaz de resolver situaciones y problemas que pueden planteársele en el ejercicio de su actividad profesional.

- Conducta (transferencia): puede definirse como el grado en que ha ocurrido un cambio en la conducta como consecuencia de haber asistido el participante a una acción formativa. Es decir, hasta qué punto aplica los conocimientos, destrezas y actitudes adquiridas en la formación. 
Para evaluar la conducta en la investigación se ha utilizado un instrumento de medición que refleja en qué grado el curso ha influido en la realidad de cada participante y usan lo aprendido en su trabajo diario (o futuro, dependiendo del tipo de curso evaluado). De esta manera tenemos en cuenta lo que Kirkpatrick denomina conducta, a través del análisis de información sobre la utilidad del curso para la realidad profesional de los participantes, lo que preferimos denominar transferencia.

- Resultados o impacto: puede definirse como los resultados finales que se obtienen como consecuencia de los participantes a un curso de formación. Los resultados finales pueden consistir en: incremento de la producción, mejora de la calidad, reducción de costes, reducción de la frecuencia y/o de la gravedad de los accidentes, incremento de las ventas, reducción de rotación de la plantilla y mayores beneficios.

Sin embargo, en educación y formación, podemos identificar los resultados o el impacto con indicadores que evalúan diferentes tasas de abandono, retraso, uso de herramientas y materiales, con el fin de conocer el porcentaje de matriculados en el curso, de personas que finalizan el mismo, de la participación de los alumnos... Dicho cambio en la evaluación de impacto por la evaluación de la ejecución ${ }^{2}$ es debida a la importancia que posee el tener en cuenta este último tipo de evaluación para el buen funcionamiento del programa formativo en su proceso de aplicación. Así, los datos referentes al impacto que puedan ser medidos, se tendrán en cuenta junto a los de transferencia mediante el instrumento de medida diseñado a tal fin.

Además de estos niveles clásicos de evaluación, hemos considerado preciso incluir otro nuevo nivel, nos referimos a la evaluación del diseño del curso en sí mismo. En esta época en la que la calidad educativa está adquiriendo gran importancia, no sólo es necesario evaluar el desarrollo y los resultados de los programas de formación tras ofertarlos al mercado, sino además tener productos sobre los que tengamos certeza de su "calidad" antes de su difusión, es decir, productos que cumplan con determinados estándares de calidad, que evalúen su adecuada puesta en marcha. Para ello, hemos creado y aplicado un instrumento de medición que evalúa las características internas del programa de formación a través de tres tipos de aspectos fundamentales de los mismos, los cuales son ya mencionados por Marqués (2000):

- Aspectos funcionales: dichos aspectos hacen referencia a la eficacia, eficiencia y relevancia del programa formativo, entre otros. 
- Aspectos técnicos y estéticos: en este apartado se consideran cada uno de los elementos que integran el programa de formación.

- Aspectos pedagógicos: en este caso pretendemos evaluar la calidad del programa formativo basándonos en indicios orientados al proceso de enseñanza-aprendizaje, centrándonos sobre todo en el impacto educativo del programa en el usuario del curso.

Para que vayamos haciéndonos una clara imagen del proceso de evaluación que hemos llevado a cabo y que se basa en una combinación de los niveles de evaluación de Kirkpatrick y de los aspectos de evaluación de Pere Marqués, una representación gráfica del mismo sería:

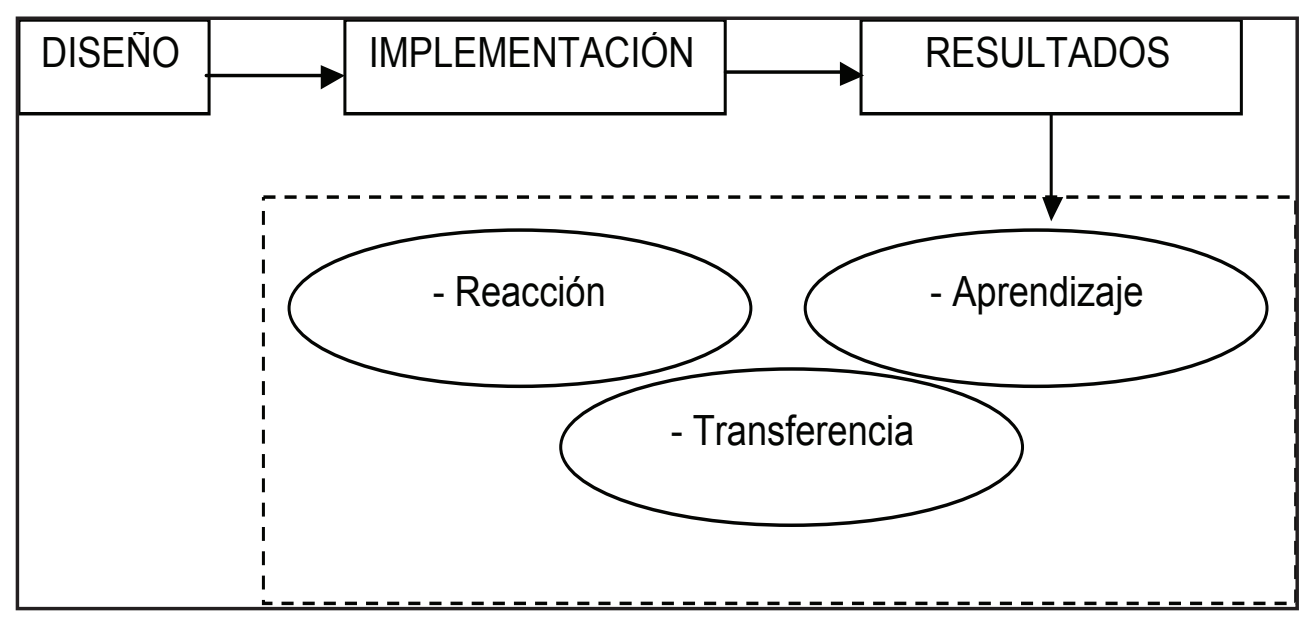

Figura 1. Proceso de evaluación a llevar a cabo

Por lo tanto, uniendo y relacionando los cinco niveles objeto de evaluación, obtendríamos el siguiente modelo de evaluación de la calidad de los cursos formativos impartidos a través de Internet (MEPFI). 


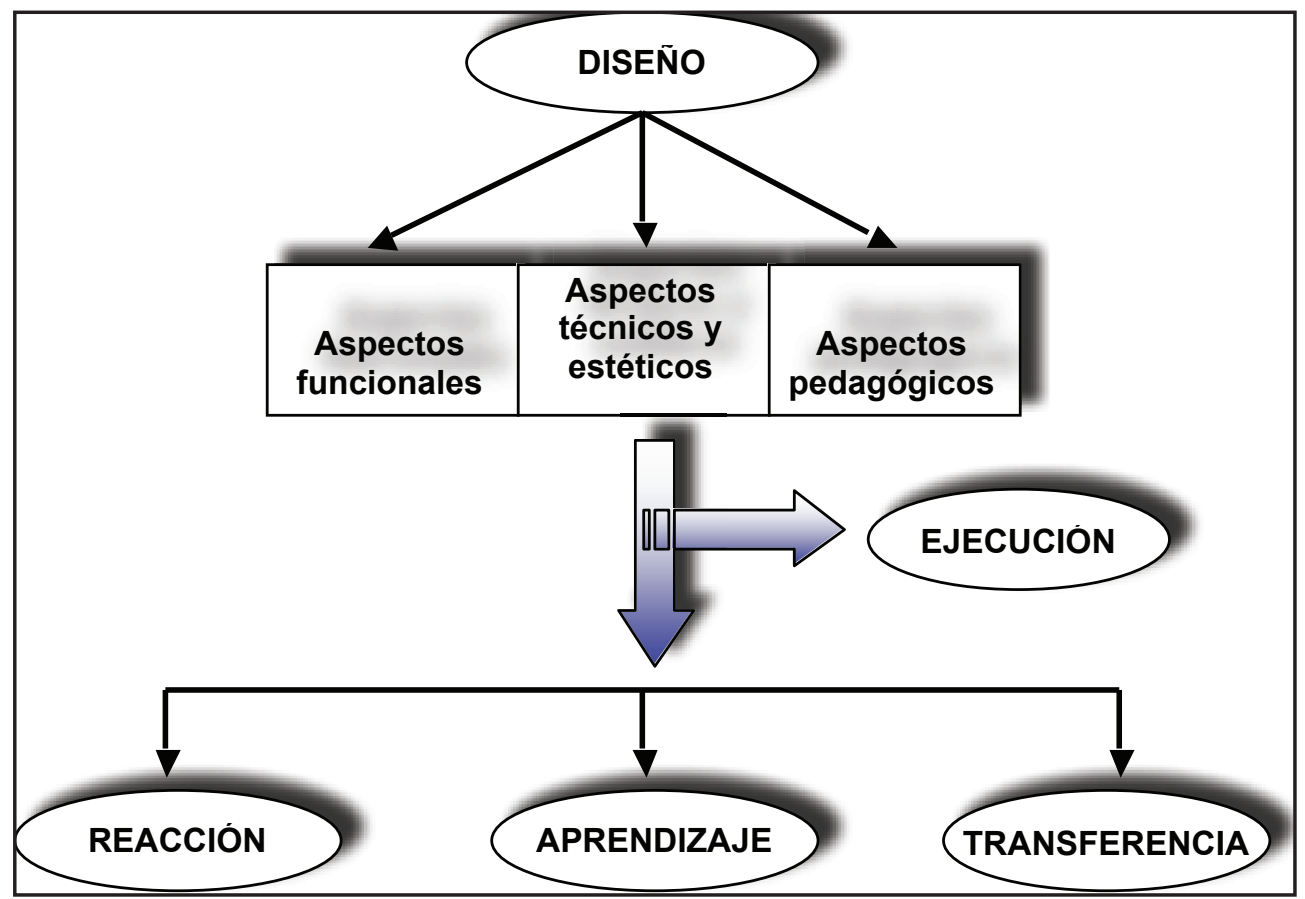

Figura 2. Modelo de evaluación de cursos formativos impartidos a través de Internet (MEPFI)

Pronto se observó que con evaluar estos aspectos de los cursos no bastaban, sino que debíamos utilizar la información que nos proporciona dicha evaluación para la mejora continua de la formación ofertada. No debemos caer en el error, bastante frecuente, de evaluar por el mero hecho de evaluar; debemos utilizar la información que nos proporciona la evaluación para incluir todas las mejoras posibles en el producto evaluado. Posterior a esta investigación han surgido otras con este objetivo de mejora, sirva a modo de ejemplo la investigación dimEDUtic: Problemas Educativos, soluciones TIC, planes de mejora (2008-2012) ${ }^{3}$ que actualmente está teniendo lugar y trata no sólo de analizar los problemas del sistema educativo, sino también de buscar soluciones TIC y aplicarlas al ámbito educativo.

Con el objeto de la mejora de los cursos evaluados, dicho modelo de evaluación de programas a través de Internet se incluyó en un ciclo sistémico de diseño, creación, aplicación, evaluación y modificación de la formación (figura 3), el cual se encuentra principalmente dividido en dos fases complementarias en el tiempo, pues van intercalando sus funciones: 
- Fase de creación, aplicación y modificación.

- Fase de evaluación del diseño, ejecución y post-ejecución.

Se ha dividido así dicho ciclo para diferenciar de forma clara y concisa aquellas funciones del modelo de evaluación mencionado anteriormente, de aquellas funciones propias del ciclo sistémico en el que se debe encontrar inmersa dicha evaluación. De esta forma, en la zona de la izquierda de la figura 3 podemos encontrar todas las funciones de la primera fase y, en la parte de la derecha de dicha figura, todas las funciones de la fase de evaluación.

Posteriormente, para el análisis de cada uno de los componentes del modelo se seguirá el orden de aplicación de cada uno, comenzando por el análisis del nivel de Diseño, posteriormente el de Implementación y, finalmente, el de Resultados. 


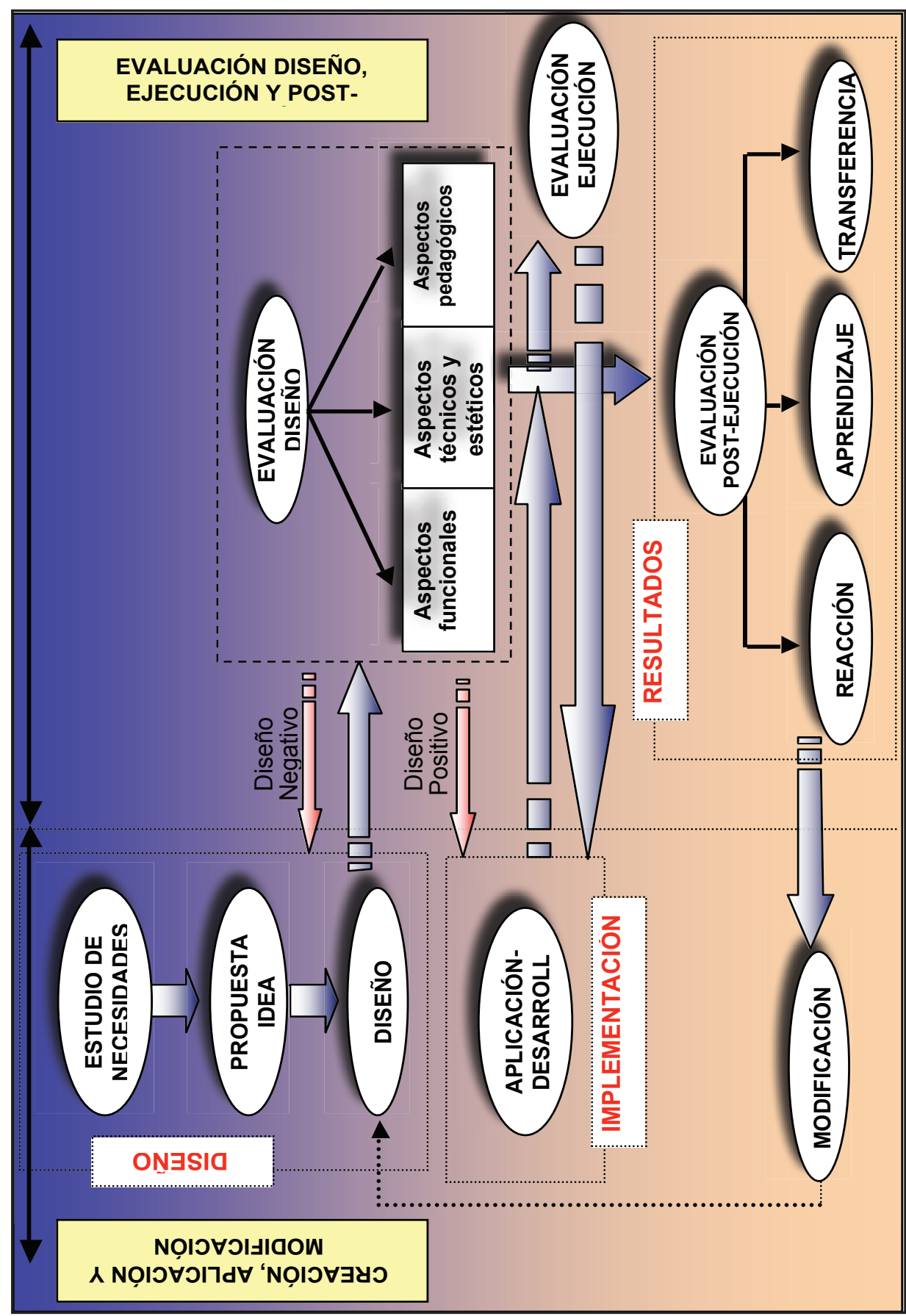




\section{DISEÑO}

\section{Estudio de necesidades de formación}

Una de las primeras cuestiones que debemos plantearnos a la hora de diseñar un plan de formación (ya sea a través de una metodología u otra), es ante qué necesidades nos encontramos. Si diseñamos y proporcionamos un producto, sin saber de antemano si va a responder a las demandas existentes, seguramente el producto creado no sea adecuado. Por lo tanto el primer paso a tener en cuenta es realizar un análisis de necesidades de formación en la institución o en el mercado, así ya lo mantenía Sparhawk (1998), cuando afirmaba que "la detección de necesidades de formación es el primer paso en el camino hacia una formación eficaz”.

Cuando en cualquier organización/institución o sociedad nos referimos a las necesidades de formación, estamos refiriéndonos al desfase existente entre las capacidades y competencias reales de los trabajadores y aquellas que requieren para la realización adecuada de su labor profesional. En este sentido, Solé y Mirabet (1994) afirman que una necesidad de formación es todo déficit observable de competencias que impide a la empresa (institución o sociedad) alcanzar sus objetivos.

Queda patente, pues, la relación existente entre las necesidades y las demandas y expectativas. Por ello, debemos tener en cuenta, de forma general, qué demandas realiza la sociedad en la que vivimos y, en particular, la realidad de la organización, así como las expectativas tanto de los directivos como de los usuarios más directos de los planes de formación creados a partir del estudio de necesidades.

De todo ello se desprende la gran importancia que hay que otorgarle a la definición y consecución de objetivos, así como los medios que necesitamos para conseguirlos.

\section{Propuesta idea}

Una vez realizado y analizado el estudio de necesidades, podemos proponer el plan de formación que vamos a llevar a cabo. Éste va a estar compuesto por ciertos cursos de formación orientados a solventar las necesidades detectadas en nuestros destinatarios, que requieren de ciertos pasos a seguir para que resulten de calidad.

Por ello, una vez que se proponen los cursos a llevar a cabo en el plan de formación, debemos pasar a diseñar cada uno de éstos, por lo que los pasos indicados a continuación deben aplicarse a cada curso o programa. 
Debemos tener en cuenta, eso sí, que la idea de plan de formación propuesta, así como los cursos que la componen, deben responder a las necesidades de, la sociedad en general, de la institución más específicamente y de los destinatarios, en particular.

\section{Diseño}

Una vez que están concretados los cursos o programas, debemos diseñarlos. El proceso de diseño de un curso es bastante laborioso y aún más si se trata de un curso semipresencial o totalmente on-line, como es nuestro caso, ya que se trata de una metodología, aún hoy en día, nueva. Aún así el diseño y la creación de este tipo de cursos es más laborioso el primer año que se pone en marcha, pues consistiría en adaptar los contenidos de un curso presencial a este nuevo formato, o crearlo totalmente en esta metodología. Y también es cierto que una vez creado es más fácil mantenerlo, modificarlo y, sobre todo, actualizarlo.

\section{Evaluación del diseño}

Como ya hemos mencionado, el propósito esencial de la evaluación que nos planteábamos al realizar esta investigación, se basaba en la evaluación de los cursos en distintos momentos de su desarrollo, de forma que se puedan detectar a tiempo los posibles inconvenientes existentes para su inmediata modificación.

De este modo, se ha decidido que en el proceso la primera evaluación que debemos realizar es la del diseño del curso. Una vez diseñado éste, debemos comprobar que se adecua a los objetivos con los que se ha creado. Para ello, se utiliza una muestra de expertos que proceden a evaluar los aspectos funcionales, técnicos y estéticos, y pedagógicos del curso.

\section{IMPLEMENTACIÓN}

\section{Aplicación/Desarrollo}

Si la evaluación del diseño del curso es positiva, podemos aplicar el programa de formación, podemos llevarlo a cabo. Si por el contrario, dicha evaluación resulta negativa, deberemos analizar las posibles causas y modificar aquellos aspectos del curso que resultan mejorables desde el punto de vista del grupo de expertos ${ }^{4}$. 
De este modo, es interesante, realizar un registro de la información del curso desde su inicio: fechas en las que comienza, tutores y profesores de cada módulo o tema, interacciones, carga de trabajo de los alumnos, apoyo técnico, resolución de problemas, posibles bajas de alumnos... Es decir, debemos dejar constancia escrita de todo el proceso de implantación del curso, para poder rectificar sobre la marcha si fuera necesario, para que no disminuya la calidad del programa formativo.

\section{Evaluación de la ejecución}

El hecho de que se esté llevando a cabo el programa de formación, no quiere decir que nos olvidemos de la evaluación hasta su terminación. Por el contrario, debemos realizar una evaluación continua del curso de formación que se está impartiendo, recogiendo datos acerca de su ejecución: número de inscritos, número de matriculados, número de bajas, causas de las bajas, conexiones, entrega de actividades, interacciones, comunicación, problemas técnicos y académicos...

Ello nos va a permitir realizar un seguimiento personalizado de los alumnos, conociendo sus necesidades, dificultades y posibles problemas con el curso y con la plataforma, personalizando así la formación. No debemos pensar que una vez iniciado el curso, no se pueden realizar cambios, al contrario, todo tipo de cambio que sea beneficioso para su adecuada ejecución, deberá ser introducido.

\section{RESULTADOS}

\section{Evaluación post-ejecución}

Una vez que el proceso de enseñanza y aprendizaje se da por concluido, se debe hacer un balance de los pros y los contras de su desarrollo.

Para ello, dividimos este momento de evaluación en tres tipos de valoración:

\section{Reacción}

Se pretende valorar el grado de satisfacción que los usuarios tienen con el curso ${ }^{5}$. De este modo, se evalúa la reacción de los alumnos ante determinados aspectos del curso, desde su inscripción y matriculación en el mismo, hasta su evaluación y calificación: facilidad de inscripción en el mismo, facilidad de uso, consecución de objetivos planteados, mapa de contenidos, adecuación delos contenidos a los alumnos, 
relevancia de las actividades, utilización de las herramientas de comunicación, guía del alumno, sistema de evaluación...

\section{Aprendizaje}

Uno de los aspectos que debemos evaluar para llevar a cabo cursos de formación de calidad, es el grado en que cambian y mejoran los conocimientos, destrezas y actitudes de los alumnos que los cursan, en definitiva, si existe un aprendizaje adecuado por parte de los alumnos. Para ello, debemos proponer un sistema de evaluación acorde con los objetivos, contenidos, metodología y actividades en las que se base el curso a lo largo de su aplicación. Este aspecto a evaluar va a depender de cada curso, por lo que en este caso no podemos crear un instrumento de medida que pueda servir para todos los cursos de metodología on-line o semipresencial, sino que dependerá de las características de cada uno.

Sí es cierto que debemos tener en cuenta que una simple nota final no mide el aprendizaje de un alumno, sino que debemos medir el aprendizaje realizado durante todo el proceso de enseñanza y aprendizaje.

En nuestro caso, debido a la gran cantidad de cursos que hemos evaluado para la realización de este trabajo, nos hemos visto obligados a basar la medida del aprendizaje de los alumnos, en el sistema de evaluación utilizado por cada uno de los responsables de los cursos, aunque nos hubiera gustado completar esta información con la extraída de un seguimiento más exhaustivo de los mismos.

De este modo, se debe tener en cuenta la importancia de evaluar de forma continua la adquisición de conocimientos y destrezas y el cambio de actitudes; lo cual servirá para introducir modificaciones en la implantación o incluir actividades de refuerzo para aprendizajes incompletos o erróneos.

\section{Transferencia}

Otro aspecto muy importante a evaluar para valorar la calidad de un curso de formación, es su posible aplicabilidad a la realidad, es decir, el grado en que los conocimientos que los alumnos han adquirido durante el curso son aplicados a la realidad. Debemos pensar que un curso es de calidad no sólo si desarrolla las competencias profesionales adecuadas en sus alumnos, sino si, además, les prepara para la aplicación de dichos conocimientos a la realidad; fuera de las aulas si se trata de formación presencial, fuera de los apuntes y trabajos si se trata de formación 
a distancia convencional y fuera de las pantallas de los ordenadores si se trata de formación a través de Internet. Y, sobre todo, a su práctica laboral activa ya sea de forma inmediata o en previsión futura.

\section{Modificación}

Como ya hemos mencionado en repetidas ocasiones, uno de los objetivos principales que nos proponemos con el modelo de evaluación que aquí se propone, se corresponde con la modificación del curso de formación evaluado para su mejora continua. Por ello, el último paso en este ciclo sistémico, antes de volver al comienzo del mismo, es la modificación del curso para, de esta forma, mejorar la calidad del mismo antes de su oferta a otros usuarios.

\section{CONSTRUCCIÓN DE INSTRUMENTOS DE MEDICIÓN PARA EL MODELO}

Una vez definido y concretado el modelo de evaluación que se va a llevar a cabo para medir la calidad de los programas formativos a través de Internet, es imprescindible concretar los instrumentos que se van a utilizar.

Para ello, debemos partir del tipo de dato que nos disponemos a recoger:

- Evaluación del diseño: llevada a cabo antes de impartir el curso de formación. Con ella se pretende recoger datos por parte de un grupo de expertos acerca de la idoneidad del programa formativo. Para ello, se crea un cuestionario con preguntas de respuesta múltiple y con preguntas abiertas. Se pretende con él recoger información acerca de la calidad de los aspectos funcionales, técnicos y estéticos, y pedagógicos del curso.

- Evaluación de la ejecución: se trata de evaluar el progreso del programa mientras se imparte, de tal forma que ante cualquier situación no beneficiosa para su funcionamiento, se pueda reaccionar modificándolo para reducir en lo posible una mala influencia en la medida de la calidad. En este momento de evaluación tendremos en cuenta, la relación entre número de inscritos y número de matriculados, el número de personas que finalizan con éxito el curso, número de bajas durante el mismo, causas de las bajas... Estos datos no serán recogidos mediante cuestionario, sino mediante la observación del funcionamiento del curso y una hoja de registro de datos e incidencias. 
- Evaluación de la reacción: una vez que finaliza el curso, es importante registrar la satisfacción de los alumnos con el mismo. De este modo, este tipo de evaluación se lleva a cabo una vez finalizado el proceso de formación y se basa en un cuestionario dirigido a los alumnos, en el que se les pregunta por su satisfacción ante diversos aspectos del curso: facilidad de uso, logro de objetivos, ritmo de trabajo, turorización, utilización de las herramientas de comunicación...

- Evaluación del aprendizaje: es interesante medir el grado de aprendizaje llevado a cabo por los alumnos al cursar el programa de formación, pues es un indicador también de la calidad del mismo. El hecho de cursar un programa formativo debe llevar consigo un aprendizaje que se muestra con la adquisición de competencias que antes de realizarlo no se tenían. Eso sí, no debemos basar la medida del aprendizaje en sólo una nota, sino realizar una evaluación continua. Al igual que ocurría con el instrumentos de medida de la evaluación de la ejecución, en este caso, tampoco se puede medir el aprendizaje mediante un cuestionario tipo, por lo que se basará dicha medida en la observación de la evolución del alumno a lo largo del desarrollo del curso.

- Evaluación de la transferencia: nos interesa además medir el grado de aplicabilidad de las competencias adquiridas con el curso a la realidad tras su finalización. Para ello, se ha creado un cuestionario de recogida de dicha información, con el que el alumno refleja su opinión sobre la utilidad de los contenidos del curso para su labor profesional, la importancia de lograr los objetivos planteados en el mismo, el tiempo en el que cree podrá aplicar lo aprendido...

Una vez planteado el tipo de dato que queríamos recoger en cada momento de evaluación, procedimos al diseño de los diferentes instrumentos de medida, los cuales pasamos a analizar en profundidad.

\section{Evaluación del diseño}

Ya hemos mencionado la importancia de evaluar el diseño de los cursos antes de ofertarlos al mercado. En este caso, se ha diseñado un instrumento que recoge la máxima información posible en torno a los aspectos funcionales, técnicos y estéticos y pedagógicos del curso. 
Como ya se ha adelantado, este cuestionario debe ser respondido por un grupo considerable de expertos (en torno a 10 como mínimo sería lo aconsejable), para detectar posibles coincidencias y diferencias en la evaluación, pudiendo llegar a un consenso sobre el resultado de evaluación del diseño de los diferentes cursos.

Este cuestionario comienza con un apartado para la recogida de datos personales del experto que contesta, puesto que se pretende tener un registro de aquellas personas que contestan a cada cuestionario con objeto de establecer posibles relaciones entre lo que responden en un curso y lo que responden a otro las mismas personas. Aún así, se respeta el anonimato de cada persona, tanto en este cuestionario como en todos los demás, ya que para identificar a las personas que contestan se utilizan las cinco últimas cifras de su DNI.

Dos de las preguntas que se incorporan en este bloque de ítems, hacen referencia a las características del ordenador desde el que los usuarios o expertos siguen o evalúan el curso, y al tipo de conexión que utilizan. Estas preguntas se han incorporado con el objetivo de analizar si una posible valoración negativa en la velocidad de navegación, funcionamiento de enlaces..., puede ser debida a las características del ordenador o al tipo de conexión a Internet desde los que se sigue el curso y no al curso en sí mismo.

Una vez recogidos estos datos personales se procede a contestar al cuerpo del cuestionario, el cual se divide en tres grandes apartados, con ítems que miden cada uno de los tres aspectos evaluados (funcionales, técnicos y estéticos y pedagógicos).

Al final de cada uno de dichos bloques se incorpora siempre una pregunta de valoración general, y una pregunta de carácter abierto para registrar posibles aportaciones que quieran realizar las personas que contestan al cuestionario.

Veamos las preguntas que conforman el cuestionario por grupos, según los aspectos que miden, para justificar por qué han sido introducidas, así como lo que se pretende comprobar con ellas, basándonos en algunas recomendaciones para mejorar la calidad de los cursos :

\section{Aspectos de la plataforma}

Es importante para evaluar los aspectos que conforman un curso de formación a través de la red, tener en cuenta la adecuación de la plataforma a las necesidades de los alumnos. Si esta no es adecuada, los usuarios no estarán satisfechos con ella y esto repercutirá en el funcionamiento adecuado del curso, ya que el soporte del mismo 
es la plataforma. Eso sí, debemos diferenciar en todo momento las características dependientes del diseño del curso de las del diseño de la plataforma, pues en algunos cursos se confunde.

Además, debemos tener en cuenta que en las plataformas on-line, la pantalla y opciones del profesor es distinta a la pantalla y opciones de los alumnos, por lo que debemos evaluar ambos perfiles de entrada en la plataforma.

En primer lugar, es importante que evaluemos la familiaridad de cada uno de los iconos de los que se compone la plataforma. No debemos caer en el error de superponer lo estético a lo didáctico. Un botón que por diseño sea muy novedoso y llamativo, pero que el alumno no sea capaz de identificar con la función para la que ha sido creado, no hará otra cosa que confundir. Debemos facilitar al alumno en lo posible el trabajo con la plataforma, para que no se le haga más trabajoso que la metodología presencial, si no el número de abandonos aumentará considerablemente.

En cuanto al correo electrónico ocurre algo parecido, ya que el alumno está acostumbrado, por lo general, a utilizar un formato de correo electrónico similar al de Outlook Express. Por ello, debemos, en lo posible, asemejar el correo de la plataforma al utilizado por el alumno de forma diaria, con un diseño fácil y funcional.

En cuanto al chat y el foro, debemos tener en cuenta que, aún hoy en día, son menos utilizados, por lo general, que el correo. De ahí que el número de alumnos que desconozcan su funcionamiento suele ser mayor. Por ello, debemos hacer del chat y el foro dos herramientas de fácil utilización y cuya estructura sea clara para su seguimiento por parte de los alumnos.

En cuanto a la videoconferencia, no debemos caer en el error de introducirla en el curso por estética, aunque su contenido y calidad no sean la adecuada. Si se introduce en el curso de formación algún contenido a través de videoconferencia ésta debe ser transmitida con total calidad de imagen y sonido, sino estaremos cayendo en el error de malgastar una herramienta que de ser bien utilizada tiene muchas funcionalidades para mejorar la calidad de un curso.

Por su parte, la agenda personal, zona personal, el glosario, los anexos y la bibliografía, son introducidos en la plataforma como herramientas para facilitar el trabajo y organización de los alumnos. Por ello, debemos intentar adaptar su diseño y funcionamiento lo máximo a las necesidades de los alumnos, para que su utilización por parte de éstos sea sencilla y clara. 
Pero si realmente queremos medir la calidad de la plataforma utilizada, así como la adecuación a sus usuarios, no podemos olvidarnos de otros usuarios de la misma, los profesores. Como hemos dicho anteriormente, el perfil del alumno es distinto al del profesor en las plataformas de formación. No debemos olvidarnos que el hecho de que un profesor sepa utilizar bien la plataforma va a influir de forma decisiva en la calidad del curso, por lo que no sólo debemos formar al profesorado en su utilización, sino que además deberemos procurar que la plataforma facilite su trabajo y, que por el contrario, no lo complique. Para ello, debemos hacer que sus herramientas sean de fácil uso, y que no requieran de mucho esfuerzo para el profesorado, el cual, al igual que los alumnos, pueden incomodarse con la plataforma y caer en el error de no utilizar ciertas herramientas de ésta o, lo que es aún peor, utilizarlas mal.

\section{Aspectos funcionales}

No debemos sólo evaluar el curso una vez que hemos comenzado a impartirlo, como ya hemos mencionado a lo largo de este trabajo, sino que debemos empezar a evaluarlo antes de impartirlo y debemos evaluar la mayoría de los aspectos involucrados en el mismo. Por ello, debemos evaluar el curso desde el sistema de inscripción al mismo. Debemos tener en cuenta que la mera inscripción del alumno al curso le va a proporcionar a éste una ligera imagen de la calidad del mismo, ya que va a estar influido por la facilidad o dificultad de dicho proceso. Por todo ello, debemos evaluar la facilidad de uso del curso desde su inscripción hasta su finalización.

Una vez que el alumno decide inscribirse en un curso con metodología online, debe cerciorarse de que cumple con los requisitos técnicos necesarios para cursarlo, pues de lo contrario le será casi imposible seguir el curso con normalidad. Además, a lo largo del curso, será muy probable que el alumno necesite asistencia técnica en alguno de los procesos del mismo, por lo que es muy importante que el curso on-line cuente con este tipo de asistencia y, además, que sea rápida y adecuada en sus respuestas a las necesidades de los alumnos. Todo esto debe aparecer documentado en el curso, para que desde un inicio los alumnos sean conscientes de estos requisitos, así como de las ayudas técnicas que tienen a su disposición.

Una de las ventajas con las que contamos al impartir docencia on-line, es que podemos adaptarnos a las características de cada alumno de forma más cómoda. Todos los alumnos que se matriculan en este tipo de cursos no tienen los mismos conocimientos, ni las mismas preferencias a la hora de estudiar. Por ello, en la medida de lo posible, es beneficioso para el curso y para los alumnos, que contemos 
con ciertas posibilidades de adaptación al contexto. No se utilizarán los mismos iconos en la plataforma si los alumnos son universitarios que si son de educación secundaria. Y, aún más importante, no tenemos por qué obligar a los alumnos a que lean los apuntes y documentos didácticos en pantalla, también pueden imprimirlos y leerlos posteriormente en papel. Son muchas las posibilidades que podemos utilizar para que el curso se adapte mejor a las preferencias de cada alumno.

\section{Aspectos técnicos y estéticos}

Como venimos ya mencionando en más de una ocasión, no debemos supeditar lo estético a lo didáctico, por lo que debemos asegurarnos que ambos estén en concordancia en el curso.

Debemos procurar que el entorno audiovisual en el que se va a mover el alumno sea adecuado, tanto desde el punto de vista de que los colores de las pantallas sean atractivos, como desde el punto de vista de que sean legibles las letras que sobre ellos aparecen. Cuando insertamos imágenes, vídeos, sonidos..., debemos cerciorarnos, antes, de que van a ser visionados en los ordenadores de los alumnos adecuadamente. Es importante que las imágenes y sonidos sean de calidad, pero aún más importante que aquellos medios audiovisuales que utilicemos tengan relevancia para acompañar al texto al que hacen referencia. No debemos utilizar este tipo de medios sólo porque hagan más atractivos los materiales, sino porque además ayuden y aclaren a los alumnos en lo que a contenidos del curso se refiere.

Por otro lado, una inadecuada velocidad en la navegación del alumno puede ser causa suficiente para que el alumno abandone el curso. Por ello, debemos ser conscientes del tiempo que tarda en cargarse una pantalla o documento con muchas imágenes, vídeos, sonidos... No queremos decir con esto que debamos obviar este tipo de recursos, al contrario, bien utilizados pueden ser muy beneficiosos. Lo que queremos decir es que debemos asegurarnos antes de insertarlos en el curso de que su tamaño no es demasiado grande para que un alumno lo recupere en un espacio de tiempo más bien corto.

Otro aspecto importante a evaluar con respecto a la navegación es el hecho de que el alumno siempre debe saber en qué zona del curso se encuentra, ya que si un alumno se pierde en el curso habrá muchas posibilidades de que abandone. En estos casos suele ser muy útil incorporar un mapa del curso que indique en todo momento en que parte del mismo se encuentra el alumno. Es imprescindible, además, el adecuado funcionamiento de enlaces, formularios..., pues de lo contrario el alumno se desanima y no entrará en estas zonas del curso. 
En cuanto a las herramientas de comunicación que ya han sido evaluadas en el apartado de aspectos de la plataforma, no debemos olvidar que también tiene repercusión el uso que de ellas se haga en el curso. Pueden estar muy bien diseñadas y hacerse un mal uso de las mismas. Por ello, deberemos evaluar si son usadas adecuadamente y si se proporcionan orientaciones de uso sobre las mismas. En cuanto al primer factor a evaluar, recordemos que este cuestionario lo responden expertos en formación on-line antes de ofertarlo al mercado, por lo que no podremos recoger este tipo de información en esta fase de la evaluación. ${ }^{6}$ Sin embargo, los expertos si podrán responder a preguntas sobre la adecuación de las orientaciones de utilización de dichas herramientas, las cuales son esenciales incorporar, por ejemplo, en la guía de estudio o de funcionamiento.

\section{Aspectos pedagógicos}

No olvidemos que estamos evaluando cursos de formación que, al fin y al cabo, e independientemente de la metodología utilizada, deben reunir una serie de características pedagógicas.

Los cursos ofertados de forma on-line, al igual que los ofertados con otras metodologías de enseñanza y aprendizaje, deberán tener explicitados unos objetivos claros y concisos que además estén relacionados con los contenidos, actividades y evaluación del curso. Todos ellos, a su vez, deberán ser adecuados para los destinatarios del curso, estar elegidos adecuadamente y contar con cierto grado de actualidad, originalidad y creatividad.

Es muy importante la presencia de la guía de estudio y la guía de funcionamiento en este tipo de cursos. La primera ayudará al alumno a seguir un curso con esta metodología, le centrará en los objetivos a lograr, le guiará por lo contenidos, le aclarará posibles dudas ya repetidas en otras ocasiones... Por su parte, la guía de funcionamiento, le proporcionará ciertas nociones del uso adecuado de las herramientas de la plataforma, así como aclaraciones de cómo abrir los materiales, como guardar documentos... Además de evaluar la existencia o no de ambas guías, debemos evaluar la adecuación de las dos a los destinatarios y la facilidad de uso.

Pero como en otras ocasiones no debemos olvidarnos de las necesidades del profesor. Él también necesita una guía que le aclare tanto aspectos de la plataforma como que le proporcione orientaciones sobre cómo impartir el curso con esta metodología. 


\section{Evaluación de la ejecución}

En cuanto a la evaluación de la ejecución del curso, no se ha creado un instrumento propio de recogida de datos, sino más bien una ficha de registro, en la que se vuelca información referente al funcionamiento del curso. Esta ficha debe ser utilizada antes, durante y tras la finalización del curso, dependiendo de la información a registrar. Por ejemplo, la información referente a inscripciones y matriculaciones, deberá ser registrada al inicio del curso, mientras las causas de las posibles bajas se irán registrando a lo largo del curso, y el número total de bajas no lo podremos tener hasta su finalización total.

A través de esta ficha podremos obtener información sobre inscripciones, matriculaciones y bajas de los alumnos y, sobretodo nos aportará mayor información sobre las posibles causas de estas bajas y problemas técnicos y didácticos sufridos. Estos datos nos ayudarán a solventar posibles problemas y dificultades de los alumnos para posteriores cursos.

\section{Evaluación de reacción}

Veamos los apartados de preguntas que conforman el cuestionario para justificar por qué han sido introducidas, así como lo que se pretende comprobar con ellas, basándonos en algunas recomendaciones para mejorar la calidad de los cursos:

\section{Satisfacción con aspectos generales del curso}

Es muy importante que los alumnos estén satisfechos con aquellas características más generales del curso, para pasar a evaluar las características más específicas del mismo. Por este motivo, se le pregunta al alumno por su satisfacción con la información que tenía del mismo (respuesta a sus expectativas), con la especificidad del calendario propuesto, con la metodología utilizada, la distribución del tiempo y las guías de estudio y funcionamiento.

Aunque en ocasiones no se le otorgue la importancia que merece, es necesario comprobar que la información proporcionada en la oferta del curso es coherente con lo que luego ha resultado ser en realidad. De esta manera estaremos respondiendo a las expectativas de aquellos alumnos que se matricularon en el curso en base a la información que se les proporcionó del mismo. 
Además, es muy importante hacerle llegar al alumno un calendario, más o menos específico, con aquellas fechas más importantes del curso, como comienzo y fin de cada módulo, entrega de actividades, evaluaciones, encuentros presenciales... De este modo, el alumno no se verá sorprendido por ninguno de estos procesos, ya que estaban fijados (aunque pueda haber variaciones previo aviso) todos ellos de antemano.

Es muy importante que los ritmos de trabajo sean adecuados, para lo cual es necesario que la distribución del tiempo de cada módulo sea suficiente para evitar agobios.

Las guías de estudio y de funcionamiento ya fueron evaluadas por los expertos antes de ofertar el curso, pero es necesario además, que sean evaluadas por los alumnos que, al fin y al cabo, eran los destinatarios de las mismas. Por ello, debemos preguntarles por su satisfacción hacia ellas, así como su adecuación y facilidad de uso.

\section{Satisfacción con las infraestructuras}

En este apartado sobre todo queremos disponernos a evaluar tres componentes de los cursos on-line: la asistencia técnica, las tutorías y los encuentros presenciales, si existiesen. En este tipo de cursos se hace casi necesaria la existencia de una persona que oriente a profesores y alumnos ante cualquier problema o duda técnica que se encuentren. A su vez, la tutoría es de los aspectos más demandados por los alumnos, ya que parece ser la herramienta de comunicación más beneficiosa para los mismos. Por eso mismo, debemos tener especial cuidado en que ésta sea adecuada, rápida y creativa.

Cuando se organicen encuentros presenciales en este tipo de cursos, el propósito de éstos, así como su fecha y hora exacta, deben ser comunicados a los alumnos con anterioridad. Debemos utilizar estos encuentros para realizar aquellas actividades que requieran de la presencia de todos, dejando lo que podemos hacer a distancia para realizarlo de forma on-line. Son actividades que pueden requerir presencialidad, por ejemplo, el acto de bienvenida y facilitador de información de funcionamiento del curso, conocerse todos los implicados en el proceso de enseñanza y aprendizaje, aclaración de dudas comunes, realización de prácticas que requieran de la presencia de los alumnos... 


\section{Satisfacción con los recursos (materiales y humanos)}

Es muy importante que los medios y materiales utilizados en el curso sean adecuados. Por una parte, debemos evaluar la satisfacción de los usuarios ante ellos, quienes nos van a proporcionar una imagen referente a la satisfacción de necesidades que éstas han cubierto. Por otro lado, el hecho de que evaluemos formación on-line, no quiere decir que dejemos de evaluar al profesor. El profesor sigue existiendo en este tipo de enseñanza (a no ser que se trate de autoformación on-line), por lo que debemos evaluar su función didáctica.

\section{Satisfacción con los aspectos técnicos y estéticos}

Aunque estos aspectos han sido ya evaluados por los expertos, necesitamos conocer la opinión de los alumnos ante ellos, que sin duda nos ofrece otra visión distinta a la de los expertos. Éstos nos informaban más de las orientaciones que se proporcionaban en el curso para su utilización, así como de su familiaridad y diseño. Sin embargo, la información de los alumnos al respecto, va a ser distinta, pero complementaria, ya que ellos los evalúan teniendo en cuenta: el funcionamiento (no da errores al utilizarlo, funciona con normalidad), el diseño (es fácil de utilizar) y la utilidad (el uso de la herramienta en el curso es beneficioso para el mismo).

\section{Satisfacción con los objetivos de aprendizaje}

Una cosa es que los expertos evalúen la adecuación de los objetivos a los destinatarios y al curso, y otra cosa es que los alumnos evalúen en qué grado creen que se han conseguido desarrollar los objetivos planteados en el mismo. Ambas informaciones son importantes, a la primera nos deben contestar expertos en la materia, mientras que a la segunda nos deberán responder los alumnos del curso.

\section{Satisfacción con los contenidos de aprendizaje}

Con los contenidos ocurre igual que con los objetivos, siendo lo más concretos posibles, ya que puede que unos contenidos no les resulten adecuados y otros sí. De este modo, se les preguntará a los alumnos por los contenidos del curso según la estructura que sigan en éste: por módulos, por temas, por bloques...

\section{Satisfacción con las actividades de aprendizaje}

Con la evaluación de los ejercicios o actividades ocurre también algo similar. El alumno debe evaluar en qué medida le han resultado satisfactorias las distintas 
actividades realizadas durante el curso, por lo que, en la medida de lo posible, habrá que preguntar por cada una de ellas, o agrupadas por temas, bloques...

Se ha introducido una pregunta referente a la posibilidad de dejar una actividad a medias, grabarla en el ordenador y continuar con ella posteriormente. Esta pregunta se introdujo ya que en algunos cursos no se introduce dicha opción arriesgando al alumno a perder toda la actividad debido a la imposibilidad de guardar el trabajo cada cierto tiempo. Este hecho, desmotiva lo suficiente al alumno como para abandonar la actividad cuando él ya la había realizado con anterioridad pero no pudo guardarla.

\section{Satisfacción con el sistema de evaluación}

El alumno puede comunicar si está conforme o no con el método de evaluación utilizado, así como su grado de satisfacción con él. En muchos cursos no se corresponde el nivel de trabajo con el método de evaluación, por lo que debemos procurar que esto no sea así. Es cierto que no debemos basar este enjuiciamiento sólo en la opinión del alumno, pero queda en manos del evaluador contrastar este tipo de información.

\section{En general}

La mayoría de las opiniones de los alumnos que son beneficiosas para posteriores ofertas del curso, se extraen de opiniones abiertas de éstos. Por ello, es importante dejar un espacio específico para ello, de forma que se le solicite al alumno opinión sobre lo que más y menos le ha gustado, así como poner a su disposición un apartado para posibles comentarios generalizados del curso que desee realizar.

\section{Evaluación del aprendizaje}

La evaluación del aprendizaje llevado a cabo por los alumnos varía en función del tipo de curso a evaluar. De este modo, los master basan su evaluación en una nota final, que generalmente depende de los trabajos realizados durante el mismo y de un proyecto final. Mientras, los cursos dirigidos a docentes en su gran mayoría, no utilizan una evaluación propiamente dicha, sino que utilizan un registro de asistencia, de participación y de actividades y trabajos para la evaluación de los aprendizajes. Y, finalmente, en cursos de Grado, existen diferentes criterios de evaluación, algunos basados en un examen, otros en trabajos y otros en las actividades de clase y asistencias. 
Por todo ello, no existe un instrumento propio de evaluación de los aprendizajes sino que nos basamos en el utilizado en cada curso para registrar si ha existido aprendizaje o no y en qué grado. Lo correcto, eso sí, sería llevar un registro continuo del aprendizaje de cada alumno paralelo al desarrollo del curso, para detectar posibles deficiencias, necesidades o problemas que puedan ser resueltos, pero dicho registro no podemos realizarlo nosotros como evaluadores externos.

\section{Evaluación de la transferencia}

El hecho de que los conocimientos adquiridos durante un determinado curso de formación, sean aplicados a la realidad de cada alumno es un indicador de calidad del curso evaluado. Por ello, se ha diseñado un cuestionario para la recogida de dicha información. Este cuestionario varía la formulación de los ítems dependiendo de las personas que lo contestan. Así, si se trata de alumno de un curso de Grado, los ítems se formulan en futuro, preguntándole por la posible aplicación de los conocimientos adquiridos en su futura labor profesional (se trata, por tanto, de la percepción de los alumnos de la utilidad de lo aprendido). Si, por el contrario, se trata de alumnos de Postgrado, los ítems se formulan en presente, para valorar la utilización de dichos conocimientos en su actual labor profesional.

Además, la aplicación de dicho cuestionario no se realiza de forma inmediata al finalizar el curso de formación, sino que se espera un prudente margen de tiempo para procurar a los alumnos la posibilidad de aplicar los conocimientos. Pasado dicho tiempo, se les aplica el cuestionario a través de correo electrónico para que lo contesten y lo remitan para ser analizado y contrastado.

\section{RESULTADOS OBTENIDOS Y CONCLUSIONES}

Una vez diseñados y creados todos los instrumentos necesarios para la recogida de información acerca de la calidad de los cursos de formación impartidos a través de Internet, seleccionamos una muestra compuesta por 15 de dichos cursos.

Ya que el modelo y los instrumentos de medida que éste conlleva han sido diseñados para su aplicación a cursos de diversas características en cuanto a la metodología semipresencial y on-line, se pretendían evaluar en el presente trabajo cursos con diferencias en su composición. Por ello fueron seleccionados cursos de diferentes niveles educativos (grado y postgrado) así como programas de formación continua para profesores. En concreto, se escogieron los siguientes cursos de la oferta 
de la Universidad Pontificia Comillas de Madrid, entre los cuales nos encontramos con todas las asignaturas de primero de Psicopedagogía, en concreto 10 asignaturas evaluadas en las distintas dimensiones que se estudian en esta investigación; tres masters y dos cursos de formación del profesorado universitario.

\begin{tabular}{|l|l|}
\hline \multirow{5}{*}{ NIVEL } & \multicolumn{1}{|c|}{ CURSOS } \\
\hline \multirow{5}{*}{ GRADO } & Diseño, desarrollo e innovación del currículum \\
\cline { 2 - 3 } & Estrategias de aprendizaje \\
\cline { 2 - 3 } & Procesos psicológicos básicos \\
\cline { 2 - 3 } & Diagnóstico en educación \\
\cline { 2 - 3 } & Modelos de orientación e intervención Psicopedagógica \\
\cline { 2 - 3 } & Psicología de la instrucción \\
\cline { 2 - 3 } & Educación especial \\
\cline { 2 - 3 } & Contextualización histórica y política de la Psicopedagogía \\
\cline { 2 - 3 } & Técnicas de Consejo Psicopedagógico \\
\cline { 2 - 3 } & Métodos, diseños y técnicas de investigación en Psicología \\
\hline \multirow{5}{*}{ FOSTGRADO } & Master y especialista universitario en migración a distancia \\
\cline { 2 - 3 } & Master on-line en mercados financieros \\
\cline { 2 - 3 } & Especialista y master en mediación familiar y con menores \\
\hline & Creación de materiales para la innovación educativa con NTIC mediante la plataforma SIFO \\
\cline { 2 - 3 } & Aplicación de las NTIC para la orientación y la tutoría \\
\hline
\end{tabular}

Tabla 1. Información académica de los cursos evaluados

El objetivo de la parte empírica de la investigación consiste en validar el modelo propuesto en la figura 2. Y, por tanto, comprobar las relaciones existentes entre las variables de evaluación: diseño, ejecución, reacción, aprendizaje y transferencia; además del análisis de las características psicométricas de los instrumentos de medida utilizados.

Hay que indicar que este objetivo nos lleva a un planteamiento que utiliza como unidades muestrales de análisis los cursos evaluados y no los sujetos que contestan a los diversos instrumentos de medida.

Recordemos llegados a este punto que las variables a medir en la investigación son varias: 


\begin{tabular}{|l|c|c|c|c|}
\hline & Previo al curso & $\begin{array}{c}\text { Durante el } \\
\text { curso }\end{array}$ & Inmediatamente & $\begin{array}{c}\text { 1 mes y medio } \\
\text { después }\end{array}$ \\
\hline Diseño & $X$ & & & \\
\hline Ejecución & & $X$ & $X$ & \\
\hline Reacción & & & $X$ & \\
\hline Aprendizaje & & & $X$ & \\
\hline Transferencia & & & & $X$ \\
\hline
\end{tabular}

Tabla 2. Variables de la investigación y momentos de medida de las mismas

No todos los aspectos son evaluados con cuestionarios, pues en el caso de los datos de ejecución y aprendizaje, han sido utilizados como instrumentos de medida una ficha de recogida de información y la entrevista con la dirección de los cursos, respectivamente.

Cabe destacar además, que el tratamiento de valores perdidos se ha realizado mediante el método Media de la serie ${ }^{7}$, con la finalidad de perder la menor cantidad de información posible.

Se debe tener presente en todos los análisis realizados, que la unidad muestral de nuestro estudio es cada uno de los cursos evaluados (15 en total, 14 tras anular uno de ellos). De este modo, se ha procedido a trabajar con 46 matrices de datos de SPSS indicadas en la figura 4.

De estas matrices, las utilizadas para la validación de los cuestionarios de la presente investigación, han sido las que toman como unidad muestral del estudio los diversos cursos, pues nos interesa precisar la utilidad y efectividad de estos instrumentos de evaluación y del modelo en sí mismo, para la mejora de la calidad de la formación on-line. Además, se han utilizado las que utilizan como unidad muestral a los diversos alumnos de los cursos, para la redacción de informes de evaluación que han sido facilitados a la dirección de cada curso con la intención de participar en la mejora de la calidad de cada uno de ellos y la matriz de datos total para la validación del modelo de evaluación. 


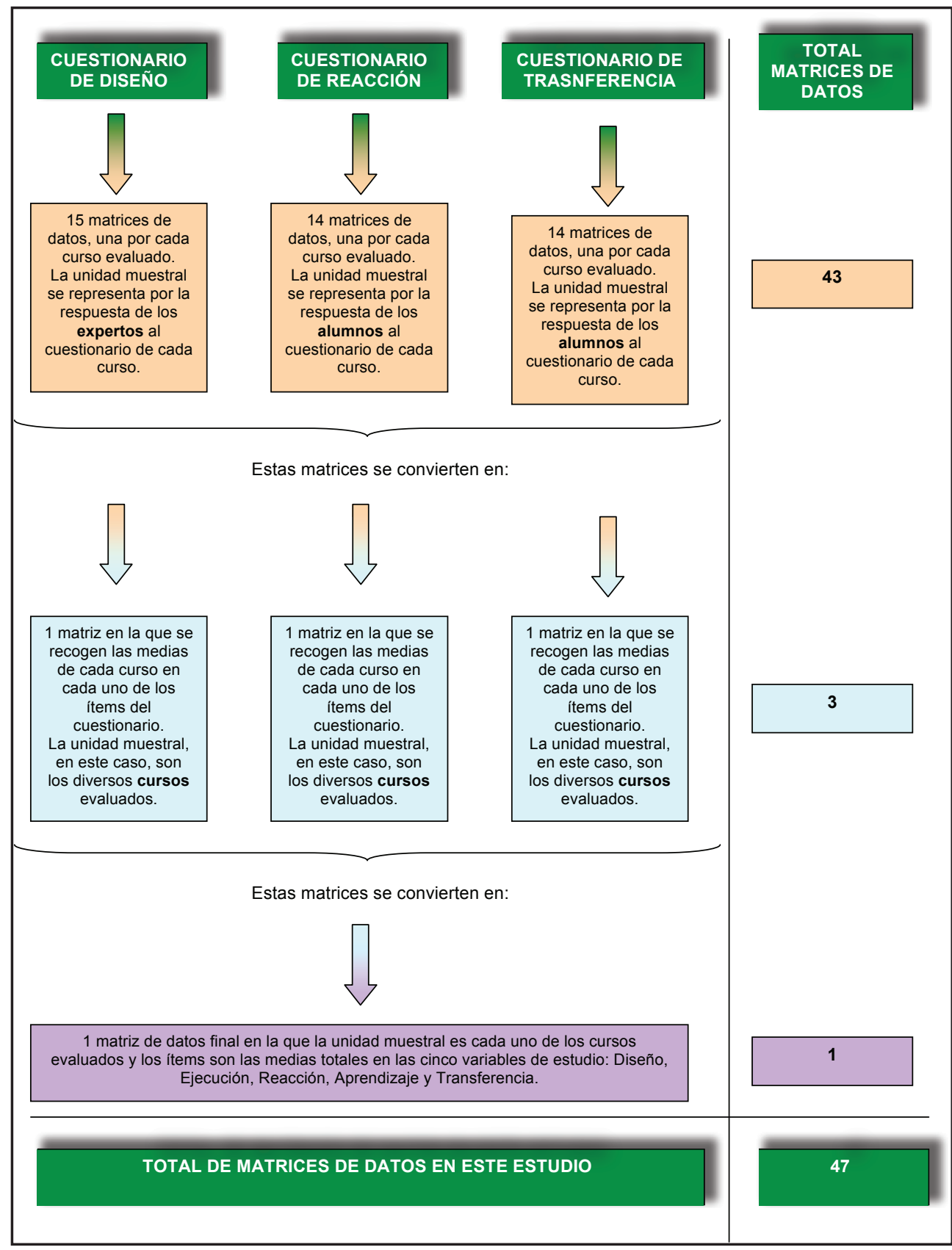

Figura 4. Distribución de las matrices de datos para los análisis estadísticos del estudio 
Como ya hemos adelantado, a partir de los datos obtenidos en los diferentes instrumentos de medida utilizados, se diseñó una nueva matriz de datos con el objeto de analizar las relaciones existentes entre las cinco grandes variables del modelo de evaluación propuesto. Las relaciones que cabría esperar a partir del modelo son las que se muestran en el gráfico 1.

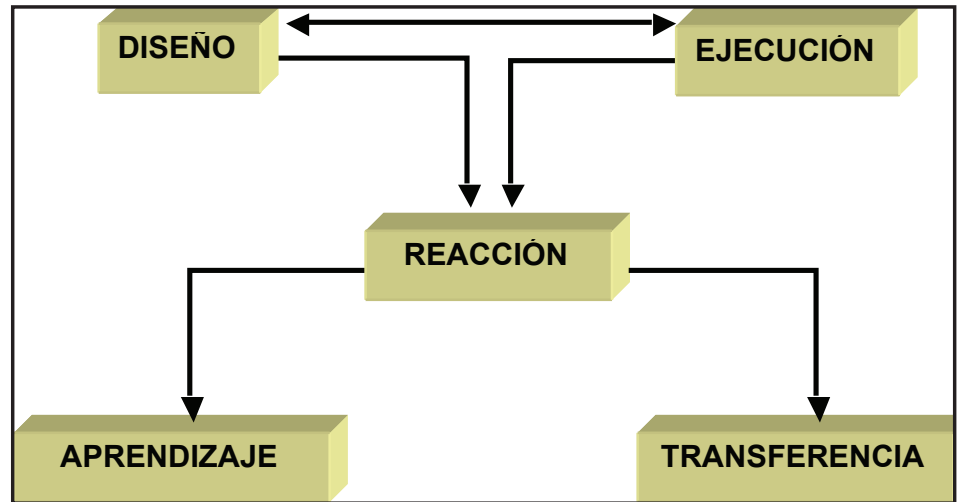

Gráfico 1. Hipótesis principal del modelo de evaluación

Como se puede observar en el gráfico 2, se han encontrado relaciones estadísticamente significativas (utilizando entre las correlaciones bivariadas el estadístico r de Pearson) entre la variable "Reacción" y las variables "Aprendizaje" y "Transferencia". Esto parece indicar que cuanto mayor es la satisfacción de los alumnos con el curso de formación a través de Internet, mayor es el aprendizaje que llevan a cabo y mayores son las expectativas de transferencia a la realidad de los conocimientos adquiridos por parte de los alumnos.

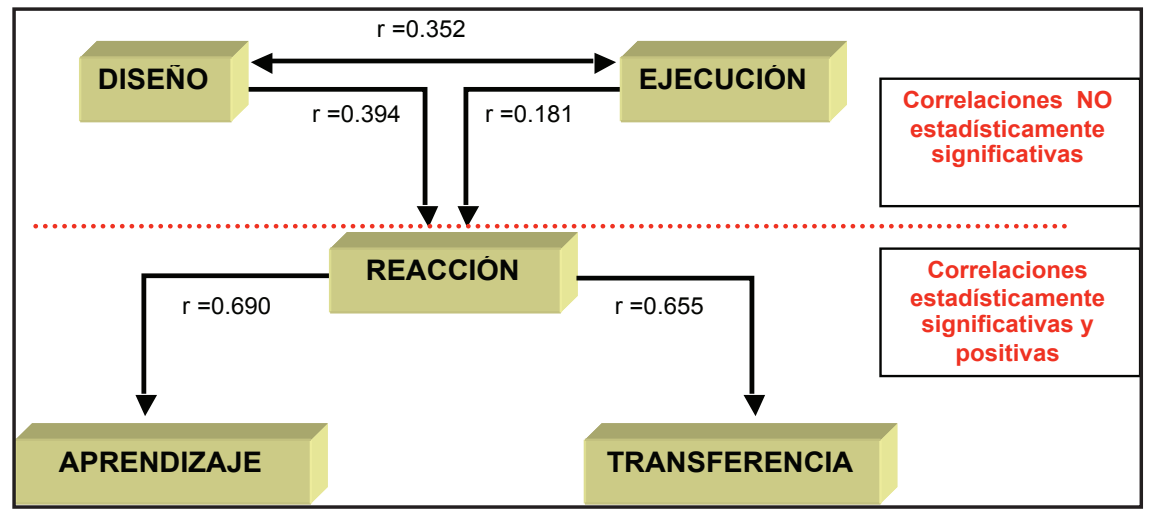

Gráfico 2. Validación de la hipótesis principal del modelo de evaluación 
El hecho de que las relaciones entre las variables diseño, ejecución y reacción no sean significativas cuando cabía esperar que lo fueran nos hizo pensar en el porqué. A pesar de que las correlaciones obtenidas de 0.352 y 0.394 podrían haber sido significativas con más muestra y la de 0.181 si se hubiese medido la variable ejecución de forma más exhaustiva, nos planteamos analizar las cinco variables, observando que aquellas entre las que se han obtenido relaciones estadísticamente significativas son las que involucran la figura del alumno: Reacción (medida a través de un cuestionario que respondía el alumno), Aprendizaje (medida a través de las notas finales del alumnado) y Transferencia (medida a través de otro cuestionario al que respondía también esta muestra de sujetos).

Se puede aprecia también cómo las variables ajenas al alumnado: Diseño (medida a través de un cuestionario al que respondía un grupo de expertos) y Ejecución (información proporcionada por la dirección del curso) no correlacionan de forma significativa con ninguna otra variable.

Ante la posible explicación de que la variable ejecución no correlaciones significativamente, pudimos observar que era una variable con una mínima heterogeneidad, puesto que medía tasa de abandono y no existían grandes diferencias en cuanto a esta medida, puesto que la mayoría de los alumnos finalizaban con éxito los diversos cursos.

En cuanto a la variable Diseño no ocurría lo mismo, por lo que debía existir alguna otra explicación. Sólo había que pensar en aquello que podía diferenciar las puntuaciones de los alumnos de las de los expertos ¿qué tenían los alumnos que no tenían los expertos? Tan sólo una cosa diferenciaba la respuesta de ambos grupos de encuestados: la presencia del profesor (aunque fuese virtual).

Los resultados del estudio sugieren que el modelo ideal de formación no es un modelo totalmente on-line, sino aquel que va más allá de lo organizativo, aquel en el que se le otorga gran importancia al contacto directo del profesor y el alumno. Es curioso que en una investigación que trata de analizar la calidad de diversos programas formativos que se imparten a través de Internet, una de las conclusiones centrales extraídas sea la gran importancia que la figura del profesor sigue teniendo y tendrá en la educación.

"Un profesor trabaja para la eternidad: nadie puede decir dónde acaba su influencia."

(Henry Brooks Adams). 


\section{NOTAS}

1 Aunque la primera edición del modelo de Kirkpatrick fue en 1944, en la bibliografía se ha utilizado la edición del 2000.

2 La cual analizaremos más detenidamente en el apartado siguiente.

3 Puede encontrarse más información sobre esta investigación en: http://www.pangea. org/dim/dimedutic/ (Consulta realizada el 20 de febrero de 2009).

4 De ahí que en el ciclo sistémico de diseño, creación, aplicación, evaluación y modificación de la formación (figura 3), nos encontremos con una flecha que vuelve al paso anterior de diseño, si la evaluación es de resultado negativo, y una flecha que concuerda con la aplicación-desarrollo del curso si la evaluación es de resultado positivo

5 Debemos recordar aquí la gran importancia que tienen las opiniones de los usuarios para lograr la calidad total en los diversos productos evaluados

6 Esta información será recogida a través del instrumento de medida referente a la reacción de los alumnos, los cuales sí pueden realizar este tipo de evaluación.

7 Este método lo que hace es que asigna a las respuestas omitidas de los sujetos, el valor medio del resto de los sujetos que contestan a ese ítem. Se puede ampliar información en: Morales, P.; Urosa, B. y Blanco, A. (2003).

\section{REFERENCIAS BIBLIOGRÁFICAS}

Galvis, A. H. (1993). Evaluación de materiales y ambientes educativos computarizados, Informática Educativa, 6, 1, (9-27).

Kirkpatrick, D. L. (2000). Evaluación de acciones formativas. Los cuatro niveles. Barcelona: Gestión 2000. (Traducción del original en inglés: Evaluating Training Programs. The tour levels. San Francisco: Berrett-Koehler. 1998).

Marqués, P. (2000). Elaboración de materiales formativos multimedia. Criterios de calidad. [en línea] Disponible en:http://dewey.uab.es/paplicada/htm/ papers/Paper_materialesmultimedia_ marques.htm [consulta 2009, 20 de febrero]

Marqués, P. (2008). Investigación dimEDUtic: Problemas Educativos, soluciones TIC, planes de mejora. [en línea] Disponible en: http://www. pangea.org/dim/dimedutic [consulta 2009, 20 de febrero]

Scheuermann, F.; Barajas, M. (2003). Aspectos pedagógicos de la enseñanza y el aprendizaje en la red. En: Barajas, M. (Coord) (2003). La tecnología educativa en la enseñanza superior. Entornos virtuales de aprendizaje. Madrid: McGraw Hill.

Solé, F.; Mirabet, M. (1994). Cómo confeccionar un plan de formación en una empresa. Barcelona: Hogar del libro.

Sparhawk, S. (1998). Cómo identificar las necesidades de formación. Madrid: Editorial Centro de Estudios Ramón Areces. (Traducción del original en inglés: Identifying Targeted Training Needs. USA: Richard Chang Associates, Inc., 1994). 


\section{PERFIL ACADÉMICO Y PROFESIONAL DE LA AUTORA}

Inmaculada Tello. Doctora en educación y Licenciada en Psicopedagogía por la Universidad Pontificia Comillas de Madrid. Master en Diseño y Programación Web por el Centro de Estudios Informáticos. Master multimedia y Educación por la Universidad Autónoma de Barcelona. Ha impartido clases en la Universidad Pontificia Comillas de Madrid, en la Universidad Autónoma de Madrid y en la Escuela Universitaria Cardenal Cisneros de Alcalá de Henares (Madrid). Ha trabajado también en la Fundación Encuentro y en la entidad pública empresarial Red.es.

E-mail: inmaculada.tello@cardenalcisneros.es

DIRECCIÓN DE LA AUTORA:

Inmaculada Tello Díaz-Maroto

Profesora dela Escuela Universitaria Cardenal Cisneros

Avenida de los Jesuitas, 34

28806 Alcalá de Henares - Madrid.

Fecha de recepción del artículo: 30/07/09

Fecha de aceptación del artículo: 06/11/09 